\title{
Screen time and psychopathology: investigating directionality using cross-lagged panel models
}

\author{
Patricia Bado ${ }^{1}$ (1) - Julia Schafer ${ }^{1} \cdot$ Andre R. Simioni $^{1} \cdot$ Rodrigo A. Bressan $^{2,3} \cdot$ Ary Gadelha $^{2,3} \cdot$ Pedro M. Pan ${ }^{2,3}$. \\ Eurípedes C. Miguel ${ }^{2,4} \cdot$ Luis A. Rohde ${ }^{2,5}$. Giovanni A. Salum ${ }^{1,2}$
}

Received: 1 July 2020 / Accepted: 24 October 2020 / Published online: 11 November 2020

○) Springer-Verlag GmbH Germany, part of Springer Nature 2020

\section{Introduction}

Children and adolescents are likely using more digital screens during the COVID-19 pandemic, raising parental concern over digital screens effects on mental health. Previous evidence on the topic has been controversial: some studies have associated more screen time with lower wellbeing in children and adolescents [1], whereas other studies found no convincing evidence that spending time on digital screens might be harmful [2]. Furthermore, this effect has been found mostly in girls than in boys [3].

However, the available evidence is mostly from cross-sectional studies, preventing investigators from understanding the directionality of such associations and excluding reverse causality, i.e., that increased levels of psychopathology lead to increased screen time. Our study investigates the bidirectional associations between screen time and psychopathology (general, internalizing and externalizing), using a

Electronic supplementary material The online version of this article (https://doi.org/10.1007/s00787-020-01675-5) contains supplementary material, which is available to authorized users.

Patricia Bado

bado.patricia@gmail.com

1 Hospital de Clínicas de Porto Alegre (HCPA), Universidade Federal Do Rio Grande Do Sul (UFRGS), Rua Ramiro Barcelos, 2350/2202, Porto Alegre, Rio Grande do Sul 90035-003, Brazil

2 National Institute of Developmental Psychiatry for Children and Adolescents (INCT-CNPq), São Paulo, Brazil

3 Universidade Federal de São Paulo (UNIFESP), São Paulo, Brazil

4 Universidade de São Paulo (USP), São Paulo, Brazil

5 ADHD Outpatient Program and Developmental Psychiatry Program, Hospital de Clínicas de Porto Alegre, Universidade Federal Do Rio Grande Do Sul, Porto Alegre, Brazil longitudinal design using data from a large school-based sample in both boys and girls.

\section{Methods}

A total of 2511 children/adolescents from the Brazilian High-Risk Cohort for Psychiatry Disorders (BHRCS) and their parents were assessed in two timepoints 3-years apart (baseline and follow-up). The BHRCS is a school-based cohort from the cities of São Paulo and Porto Alegre, with one subgroup selected based on a high-risk score for psychiatric disorders $(n=1554)$ and another subgroup randomly selected $(n=957)$. This study was approved by the ethics committee of the University of São Paulo (IRB registration number: 1132/08). For more information about sampling see Salum et al. [4]. Children and adolescents had a mean age of 10.4 years-old at baseline (min-max 5.8-14.4) and 13.7 (min-max 9.2-17.9) at follow-up. 1375 (54.8\%) were boys and 1256 (50.1\%) were from São Paulo while others were from Porto Alegre. Participant's characteristics are shown in Table 1.

Daily screen time (e.g. time spent in front of computer, television and others) was obtained by asking the parent or the primary caregiver "How many hours a day does the child spend in front of the television, using the computer and playing video games on average"? Responses were given in total numbers of hours. Since baseline data was acquired in 2010, mobile devices were not included. Psychopathology was measured by the Child Behavior Checklist, using bifactor models to estimate a measure of overall psychopathology (the " $p$ " factor) with two residualized dimensions of internalizing and externalizing psychopathology [5].

Data were analyzed using Cross-Lagged Panel Models (CLPM). First, we conducted factor analyses to assess the latent structure of psychopathology. We then used factor scores from measures of psychopathology to test for 
Table 1 Participant and variable characteristics

\begin{tabular}{|c|c|c|c|c|c|c|}
\hline & \multirow[b]{2}{*}{ Valid $N$} & \multicolumn{2}{|l|}{ Baseline } & \multicolumn{3}{|c|}{ 3-year follow-up } \\
\hline & & Mean (sd) & Min-Max & Valid $N$ & Mean (sd) & Min-Max \\
\hline Age (years) & 2511 & $10.2(1.9)$ & $5.8-14.4$ & 2010 & $13.5(1.9)$ & $9.2-17.9$ \\
\hline CBCL: Total CBCL scores & 2511 & $17.2(16.1)$ & $0-101$ & 2010 & $14.6(15.0)$ & 0-90 \\
\hline Screen Time (hours/day) & 2267 & $3.5(1.87)$ & $0-12$ & 1861 & $3.9(2.77)$ & $1-15$ \\
\hline
\end{tabular}

Crude scores for psychopathology (CBCL) are presented to inform about the variables' characteristics on the sample. From the total participants, $54.8 \%$ were boys and 50\% were from São Paulo

$C B C L$ Child Behavior Checklist bidirectional effects between psychopathology and screen time through CLPM in baseline and 3-year follow-up assessments. Data analysis was performed using the lavaan package from $\mathrm{R}$ [6]. Total number subjects without missing data in both time points were 1617 and missings were accounted for using full information maximum likelihood. Five subjects were excluded due to implausible values in screen time ( $>15 \mathrm{~h}$ ). Given previous evidence showed sex-differences in the association between screen time and psychopathology, we also tested whether associations varied by sex.

\section{Results}

Mean screen time was $3.41(\mathrm{sd}=1.91)$ at baseline and $3.95(\mathrm{sd}=2.66)$ at follow-up. Cross-sectional correlations between screen time and psychopathology were significant, but modest, at both time points (baseline: $r=0.103$, $p<0.001$; follow-up: $r=0.134, p<0.001)$. Cross-lagged models aiming to estimate directional effects between baseline and follow-up showed that higher overall psychopathology at baseline predicted higher screen time at follow-up $(\beta=0.144, p<0.001)$, but that screen time did not significantly predict psychopathology $(\beta=0.020, p=0.073)$, Fig. 1. Screen time was predicted by internalizing $(\beta=0.316$, $p<0.001)$ but not by externalizing symptoms $(\beta=-0.067$, $p=0.242$ ), Supplemental Fig. 1a, b. Internalizing symptoms predicted screen time significantly in both sexes $(\beta$ girls $=0.374, p<0.001 ; \beta$ boys $=0.177, p=0.034)$, although this effect was stronger in girls than in boys $(t=1.98$, $p=0.047)$. Externalizing symptoms did not significantly predict screen time in boys $(\beta=0.190, p=0.074)$ nor in girls $(\beta=-0.102, p=0.093)$.

\section{Discussion}

Our results showed that higher psychopathology at baseline predisposes to higher screen time in follow-up. Therefore, higher use of screens by children and adolescents could be a result of psychopathology, rather than a cause. Psychopathology effects on screen time were observed mostly for internalizing symptoms and for girls while externalizing symptoms were not significant. Most effect sizes detected in the study were of a small magnitude.

Other studies have called attention to the modest associations between screen time and mental well-being [2]. Previous longitudinal studies found no influence of screen time [7] or only a modest influence [8] on mental health problems. Our cross-lagged association was also modest for general psychopathology, but stronger for internalizing symptoms, especially in girls. The main finding of the current study, however, is not the association itself, but its directionality.

Our study has important strengths. First, we make use of a longitudinal design with measurements on screen time and psychopathology on both timepoints using models that can disentangle the directionality of association. Second, the sample was ascertained by the community, decreasing the change of several sorts of selection bias when participants
Fig. 1 Cross-Lagged Panel Models for bidirectional associations between screen time and psychopathology at baseline and follow-up. Beta coefficients from cross-model regressions between the two-timepoints are shown, as well as from crosslagged covariance at each time point. ${ }^{*} p<0.001, * p<0.05$

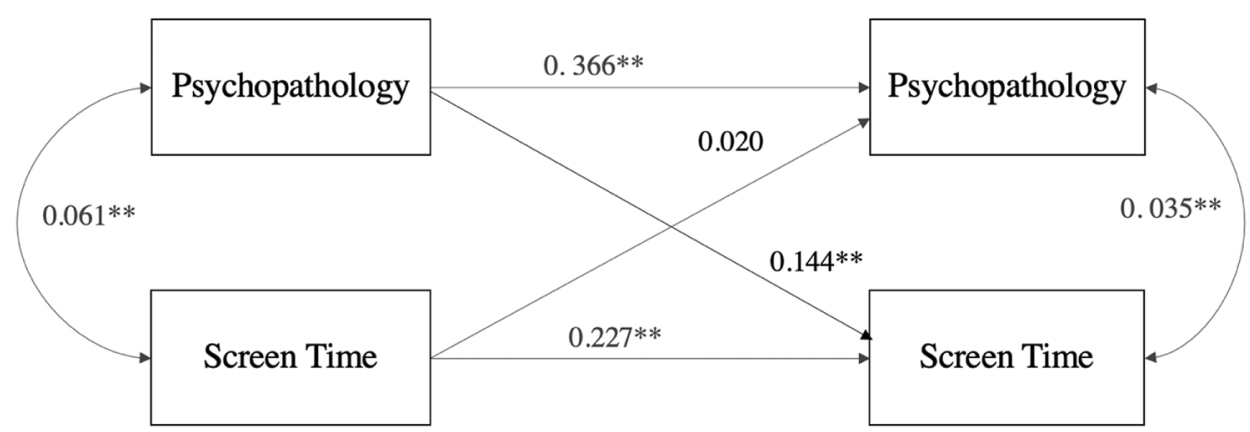


are selected by social media. However, it also has important limitations. Screen time was characterized by a single variable assessed by parental reports in a questionnaire. Furthermore, our measure is somewhat specific to time spent on television, computer and videogames and does not include other screens that were not common at the time the cohort started.

In conclusion, we demonstrate a causal association between psychopathology and screen time in the opposite direction of common sense. Public health policies on screen restriction might be ineffective by not considering this reverse causality, in which screen time could be an indicator but not a cause of psychopathology.

Acknowledgments This work is supported by the National Institute of Developmental Psychiatry for Children and Adolescents, a science and technology institute funded by Conselho Nacional de Desenvolvimento Científico e Tecnológico (CNPq; National Council for Scientific and Technological Development; grant number 573974/2008-0). This study was also financed by the Coordenação de Aperfeiçoamento de Pessoal de Nível Superior-Brasil (CAPES)_Finance Code 001 and by National Institute of Mental Health (NIMH), grant number RO1 MH120482-01, subaward nº 576811.

\section{Compliance with ethical standards}

Conflicts of interest Patricia Bado, Julia Schafer, Andre R. Simioni, Eurípedes C. Miguel and Giovanni A. Salum declare no conflict of interest. Rodrigo A. Bressan has received personal fees and non-financial support from Janssen, personal fees from Pfizer, Torrent and Sanofi-Aventis outside the submitted work; Ary Gadelha has been a speaker and/or advisor to or has received honoraria from Janssen, Daiichi-Sankyo, Aché, Cristália and Torrent; Luis Augusto Rohde has received grant or research support from, served as a consultant to, and served on the speakers' bureau of Medice, Novartis/Sandoz and Shire/
Takeda in the last three years. The ADHD and Juvenile Bipolar Disorder Outpatient Programs chaired by Dr Rohde have received unrestricted educational and research support from the following pharmaceutical companies in the last three years: Novartis/Sandoz and Shire/ Takeda. Dr Rohde has received authorship royalties from Oxford Press and ArtMed and travel grants from Shire to take part in the 2018 APA annual meeting.

\section{References}

1. Twenge JM, Campbell WK (2018) Associations between screen time and lower psychological well-being among children and adolescents: evidence from a population-based study. Prev Med Rep 12:271-283

2. Orben A, Przybylski AK (2019) The association between adolescent well-being and digital technology use. Nat Hum Behav 3:173-182

3. Booker CL, Kelly YJ, Sacker A (2018) Gender differences in the associations between age trends of social media interaction and well-being among 10-15 year olds in the UK. BMC Public Health 18:321

4. Salum GA et al (2015) High risk cohort study for psychiatric disorders in childhood: rationale, design, methods and preliminary results. Int J Methods Psychiatr Res 24:58-73

5. Schäfer JL et al (2020) Cross-sectional and longitudinal associations of threat and deprivation on cognition, emotional processing and psychopathology in children and adolescents. BioRxiv. https ://doi.org/10.1101/2020.02.09.940858

6. Rosseel Y (2012) lavaan: an R Package for structural equation modeling. J Stat Softw Artic 48:1-36

7. Jensen M, George MJ, Russell MR, Odgers CL (2019) Young adolescents' digital technology use and mental health symptoms: little evidence of longitudinal or daily linkages. Clin Psychol Sci 7:1416-1433

8. Wu X et al (2016) Impact of screen time on mental health problems progression in youth: a 1-year follow-up study. BMJ Open 6:e011533 